\title{
Intra-beam scattering and its applications to ERL
}

\author{
A. Fedotov
}

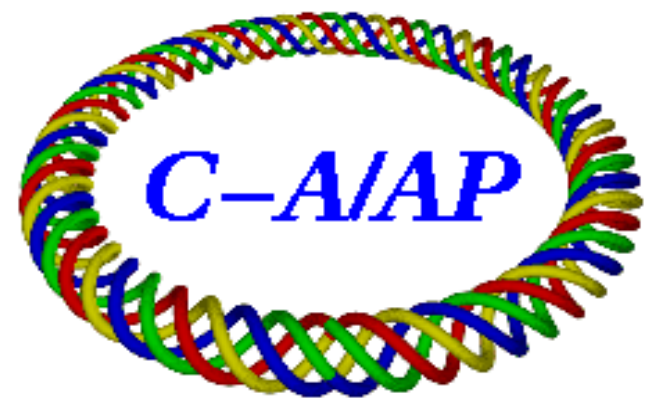

\section{Collider-Accelerator Department Brookhaven National Laboratory Upton, NY 11973}

Notice: This document has been authorized by employees of Brookhaven Science Associates, LLC under Contract No. DE-AC02-98CH10886 with the U.S. Department of En ergy. The United States Government retains a nonexclusive, paid-up, irrevocable, world-wide license to publish or reproduce the published form of this document, or allow others to do so, for United States Government purposes. 


\title{
INTRA-BEAM SCATTERING AND ITS APPLICATION TO ERL
}

\author{
Alexei Fedotov \\ Collider-Accelerator Department, BNL, Upton, NY 11973, USA
}

Presented at the 50th ICFA Advanced Beam Dynamics Workshop on Energy Recovery Linacs, KEK, Tsukuba, Japan, 16-21 October 2011. 


\title{
INTRA-BEAM SCATTERING AND ITS APPLICATION TO ERL*
}

\author{
A.V. Fedotov ${ }^{\#}$, BNL, Upton, NY 11973, USA
}

\begin{abstract}
Treatment of Coulomb collisions within the beam requires consideration of both large and small angle scattering. Such collisions lead to the Touschek effect and Intrabeam Scattering (IBS). The Touschek effect refers to particle loss as a result of a single collision, where only transfer from the transverse direction into longitudinal plays a role. It is important to consider this effect for ERL design to have an appropriate choice of collimation system. The IBS is a diffusion process which leads to changes of beam distribution but does not necessarily result in a beam loss. Evaluation of IBS in ERLs, where beam distribution is non-Gaussian, requires special treatment. Here we describe the IBS and Touschek effects with application to ERLs.
\end{abstract}

\section{INTRODUCTION}

A subject of Coulomb scattering within charged particle beams is well established in circular particle accelerators. In this report a brief summary is given with an emphasis on applications to the future high-current high-brightness Energy Recovery Linacs (ERLs). Here we do not attempt to produce a comprehensive list of references on existing IBS models but rather limit discussion to just a few with which we had some experience. Some specifics of the Touschek effect and IBS in ERLs are also discussed.

The effect when particles within the beam can be lost as a result of a single collision event (large-angle scattering) is called Touschek effect [1]. The cause of the Touschek effect is the transformation of the transverse momentum in longitudinal with its amplification by the relativistic factor $\gamma$. The particles are lost after collision if the change introduced in the longitudinal momentum is larger than the energy acceptance of accelerator.

When the scattering angles are small, random addition of such small scattering events can lead to a growth of beam dimensions. Such a multiple Coulomb scattering was first applied to explain emittance growth in electron beams $[2,3]$ and was called "multiple Touschek effect". The multiple Coulomb scattering was later generalized by Piwinski for proton machines without making any restrictions on the magnitude of beam temperatures, thus making it possible to transfer energy from the longitudinal into transverse via collisions [4]. This generalized treatment of multiple small-angle Coulomb scattering was also renamed as the Intrabeam Scattering (IBS) [4]. The IBS theory was later extended to include variations of the betatron functions and momentum dispersion function along the lattice of accelerator, and was summarized in reports by Martini [5] and Piwinski [6].

\footnotetext{
*Work supported by the U.S. Department of Energy

"fedotov@bnl.gov
}

The different approach to IBS using the scattering matrix formalism from quantum electrodynamics was used by Bjorken and Mtingwa (B-M model) [7]. Both B$\mathrm{M}$ and Martini's models are in good agreement with one another.

Note that a variety of IBS models were derived based on the original models of Bjorken-Mtingwa, Martini and Piwinski, which can produce different results, especially when used outside their region of applicability. In our experience with IBS simulations and experimental verification, exact Bjorken-Mtingwa [7] and Martini [5] models produced similar results both above and below transition energy of an accelerator.

Typical limitation of analytic models of IBS is that they are developed in an assumption of Gaussian distribution. In most situations such treatment is justified and models provide good agreement with experimental measurements (see Ref. [8], for example). However, when distribution starts to deviate from Gaussian significantly, assumption of Gaussian distribution may result in inaccurate predictions. To address this issue 1-D FokkerPlanck approach was effectively used before [9]-[10].

A more dramatic situation occurs when there is an externally applied force, like electron cooling. Since electron cooling force depends on the amplitudes of individual particles, the distribution under such force very quickly deviates from Gaussian. The problem of how to accurately account for IBS for such distributions became of special interest with a proposal to use electron cooling directly in a collider. Several approximate models were developed in the past to address this issue [11-13].

A more general description requires full treatment of kinetic problem. Such a treatment was introduced in the BETACOOL code [14] under the name "local IBS model" [15]. In addition to extensive numerical tests it was also benchmarked vs. experimental data with results reported in Ref. [16].

With application to ERLs, an approximate treatment using sliced-beam approach was suggested in Ref. [17]. In present report, a comparison between sliced-beam and local-IBS models is presented.

An analytic analysis of Coulomb scattering for a variety of distributions in 3-D was also performed in the past to understand possible halo formation in linear accelerators $[18,19]$. These studies also discussed an extent of beam halo due to such collisions. 


\section{TOUSCHEK EFFECT AND SCALING FOR MULTI-PASS ERL}

Theoretical investigation of Touschek effect in accelerator community started with assumption of flat beams and thus transfer of only horizontal momentum into longitudinal [1,3]. An assumption of non-relativistic velocities of colliding particles in their center of mass system was also used. The theory was further extended to take into account the cross-section valid for relativistic velocities [20]. The treatment was later generalized to 2-D to take into account transfer of both horizontal and vertical momentum with assumption of transversely round beam [21].

A more general treatment in 2-D for arbitrary ratios between horizontal and vertical amplitudes and arbitrary velocities was presented by Piwinski [22]. Piwinski's generalized expression produces other formulas by taking corresponding limits. This generalized expression of Piwinski was implemented in simulations codes and already applied for particle tracking in ERLs for APS [23] and Cornell [24] projects, for example.

For proposed eRHIC ERL [25] which is a multi-pass ERL with 6-pass acceleration to reach top energy and 6 passes to decelerate the beam, we are interested in the tails of loss distribution resulting from Touschek scattering. A net result for such distribution accumulated after total of 12 passes is shown in Fig. 1 for beam parameters shown in Table 1. For this plot we used 2-D expression for the scattering rate from Ref. [21].

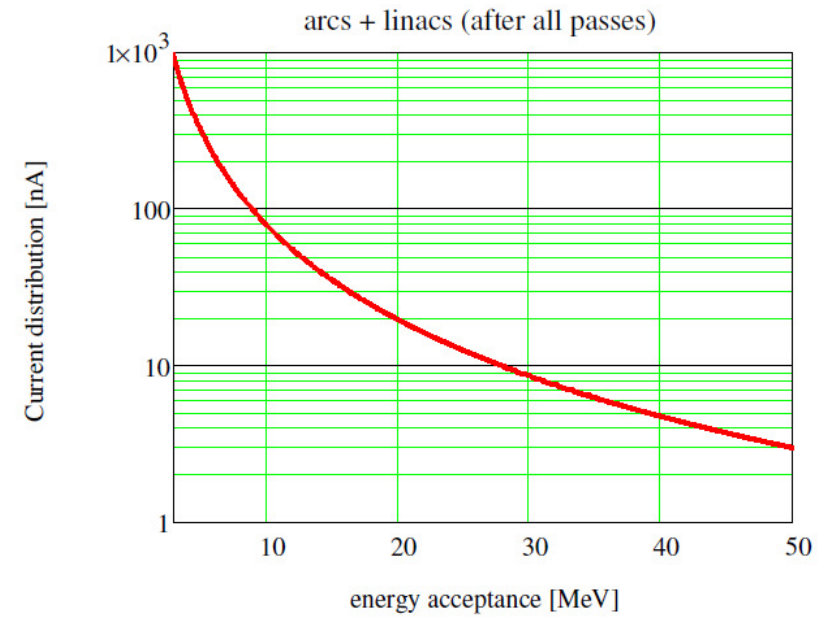

Figure 1: Resulting loss distribution from Touschek scattering after all 12 passes in eRHIC ERL expressed in terms of beam current outside energy deviation in $\mathrm{MeV}$ (zero on the axis is suppressed).

Table 1: Beam parameters used for Touschek calculations of $20 \mathrm{GeV}$ eRHIC ERL.

\begin{tabular}{|l|l|}
\hline Total length of beam transport, $\mathrm{km}$ & 46 \\
\hline Bunch charge, $\mathrm{nC}$ & 3.5 \\
\hline RMS bunch length, mm & 2 \\
\hline Normalized rms emittance, $\mathrm{mm} \mathrm{mrad}$ & 20 \\
\hline Average current, $\mathrm{mA}$ & 50 \\
\hline
\end{tabular}

In the course of these studies, the question came up whether resulting distribution in Fig. 1 is dominated by low-energy or high-energy passes in our multi-pass ERL. To understand scaling we use the same expression from Ref. [21]:

$$
\frac{1}{N} \frac{d N}{d t}=\frac{2 \pi}{\gamma^{4}} \frac{c r_{c}^{2} N F(\varepsilon)}{(4 \pi)^{3 / 2} \theta_{y} \theta_{x} \sigma_{x} \sigma_{y} \sigma_{z}\left(\Delta E_{m} / E\right)}
$$

but replace function $F(\varepsilon)$ by its approximate value

$$
F(\varepsilon)=\frac{1}{\sqrt{\varepsilon}}-6
$$

valid for small $\varepsilon$ where $\varepsilon=\left(\left(\Delta \mathrm{E}_{\mathrm{m}} / \mathrm{E}\right) /\left(\gamma \theta_{\mathrm{x}}\right)\right)^{2}$, and spread of the momentum in the horizontal and vertical directions is assumed the same (full coupling $\beta_{x}=\beta_{y}$ ). Expressing in terms of the normalized emittances one gets:

$$
\frac{1}{N} \frac{d N}{d t}=\frac{2 \pi c r_{c}^{2} N \gamma^{1 / 2}}{(4 \pi)^{3 / 2} \varepsilon_{n y} \varepsilon_{n x} \sigma_{z}\left(\Delta E_{m}\right)^{2}} \sqrt{\frac{\varepsilon_{n x}}{\beta_{x}}}
$$

where $N$ is the number of particles per bunch, $r_{c}$ is the classical radius of particle, $\varepsilon_{n x}$ and $\varepsilon_{n y}$ are transverse normalized rms emittances, $\sigma_{z}$ is the rms bunch length, $\beta_{x}$ is average of the lattice beta-function and $\Delta E_{m}$ is energy deviation in absolute energy units. Equation (3) shows how each pass contributes to the current-loss distribution from Touschek scattering, with higher energies giving the largest contribution. Such relative contribution from different energies for eRHIC is shown in Fig. 2.

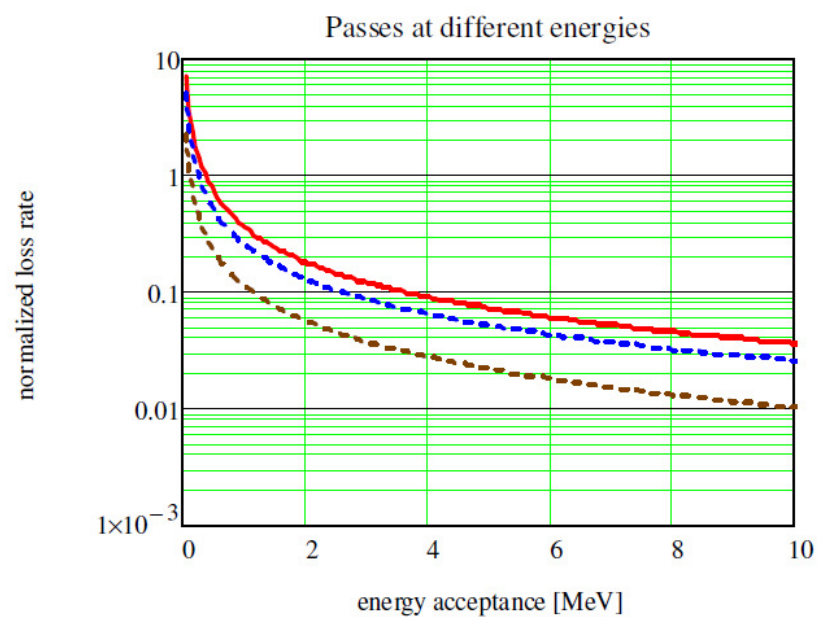

Figure 2: Relative contribution (in normalized units) to Touschek loss distribution from passes at $20 \mathrm{GeV}$ (red upper curve), $10 \mathrm{GeV}$ (middle blue curve) and $2 \mathrm{GeV}$ (brown lower curve) for the case of eRHIC. 


\section{IBS IN ERL}

In comparison to circular accelerators, very large IBS growth rates are required to get noticeable distribution change because of a very short time the bunch spends in ERL. An order of magnitude estimate of both single and multiple scattering events can be obtained using, for example [18]:

$$
\frac{1}{\tau}=K_{n} \frac{r_{c}^{2} N c}{\gamma^{2} \varepsilon_{n y} \varepsilon_{n x} \sigma_{z} \sigma_{p}} \Lambda_{c} \Lambda_{n}
$$

where $K_{n}$ is the normalization coefficient for a specific distribution function (less than unity), $\sigma_{p}$ is rms energy spread, $\Lambda_{n}$ is the logarithm needed for some singular distributions in 3-D (not needed for a Gaussian distribution), and $\Lambda_{c}$ is the Coulomb logarithm (needed only for multiple scattering) the value of which varies from 10 to 15 for typical parameters of electron beams. Using parameters of some hypothetical high-brightness ERL from Table 2, and putting $K_{n}=\Lambda_{n}=1$ for this order-ofmagnitude estimate, one gets IBS growth rates of about 1000 1/s which is large but not sufficient to cause any worry. One should note that growth rate will be significantly larger for low energies. As a result, the effect could be significant if one considers long transport of high-brightness beams at low energies.

Table 2: Beam parameters of some possible highbrightness ERL.

\begin{tabular}{|l|l|}
\hline Relativistic $\gamma$ & 1000 \\
\hline Bunch charge, $\mathrm{nC}$ & 2 \\
\hline Bunch length $\sigma_{\mathrm{z}} / \mathrm{c}, \mathrm{ps}$ & 1 \\
\hline Normalized rms emittance, $\mathrm{mm}$ mrad & 1 \\
\hline Rms momentum spread & 0.001 \\
\hline
\end{tabular}

However, it was recently pointed out that electron distribution in ERL is highly non-Gaussian, especially in the longitudinal plane. As a result, local IBS longitudinal rates can be very large due to small local longitudinal velocity spread within longitudinal slices of beam distribution. A simplified model of sliced-beam approach was suggested to treat such non-Gaussian distributions in ERLs [17].

A similar problem of IBS for non-Gaussian distribution was extensively studied in electron cooling community with a variety of approximate models developed. An approach based on amplitude-dependent diffusion coefficients was also implemented in BETACOOL code [14] with extensive numerical benchmarking and comparison with experimental data [16]. Such implementation allows one to treat IBS for arbitrary distribution in 3-D, including when distribution is affected not just by IBS but also by some other amplitudedependent force, like cooling. In the following section we present simulation comparison between such "local" IBS approach [16] and sliced-beam approximation which was also implemented in BETACOOL code [26].
Before going into discussion of simulation results based on various models, we note that large local rate within a longitudinal slice of the beam should not necessarily lead to a significant change in beam distribution. The process of IBS is described by a diffusion in the velocity space. Since intrinsic momentum spread in typical ERL distribution is very small in each longitudinal slice of the beam, the longitudinal velocity spread in beam frame is much smaller than transverse. In such a case one can show that the longitudinal diffusion coefficient becomes almost independent of the longitudinal velocity spread (see [27, 13], for example), and thus very little growth could be expected despite the fact that local longitudinal rates are very high.

\section{SIMULATIONS OF IBS FOR ERL}

For a test between "local" $[15,16]$ and "sliced-beam" approaches we use ERL beam distribution which was produced for our previous studies of high-energy electron cooling [28]. The histogram of velocities in such is shown in Fig. 3, and the longitudinal phase-space in Fig. 4.

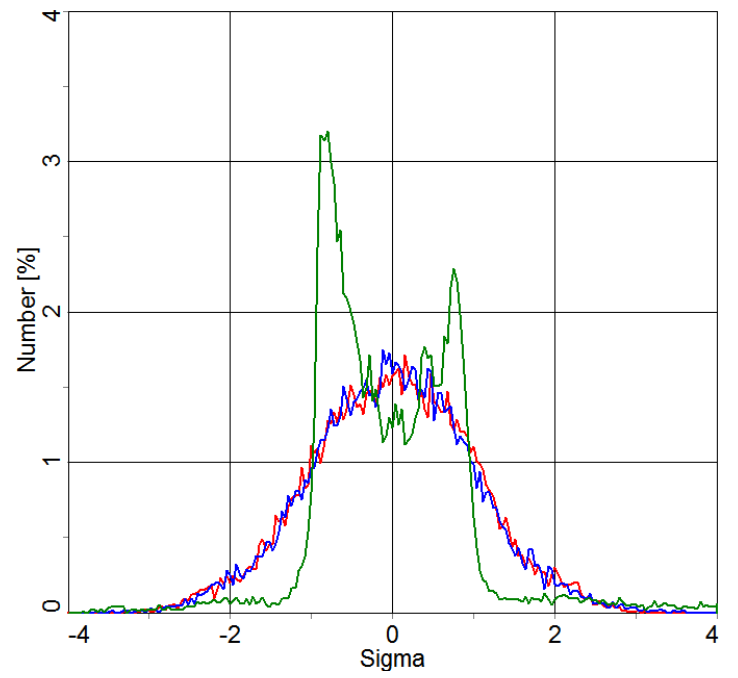

Figure 3: Histogram of velocity distribution of electrons. Red and blue - horizontal and vertical; green longitudinal.

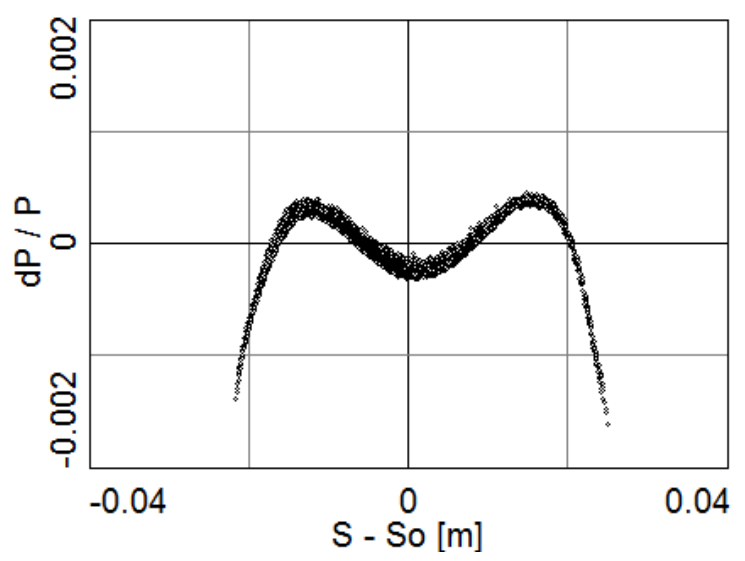

Figure 4: Initial longitudinal phase-space of distribution used in simulations. 
First, we choose such beam parameters that IBS rates calculated based on rms quantities are small $151 / \mathrm{s}$ and local rates within the slices (Fig. 5) are not sufficient to cause significant distribution change. We then track such distribution over $4 \mathrm{~km}$ of beam transport line using the BEATCOOL code. As expected, no effect is observed both with the "local" and "sliced" beam approaches, as shown in Figs. 6 and 7, respectively.

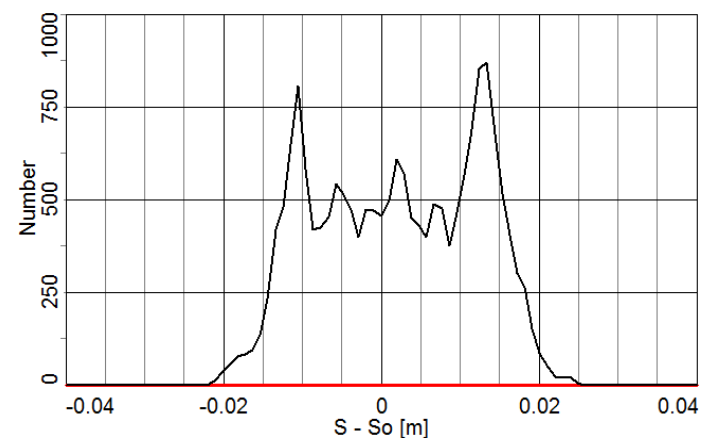

Figure 5: IBS rates within longitudinal slices of beam distribution. Vertical axis: local IBS rates [1/s].

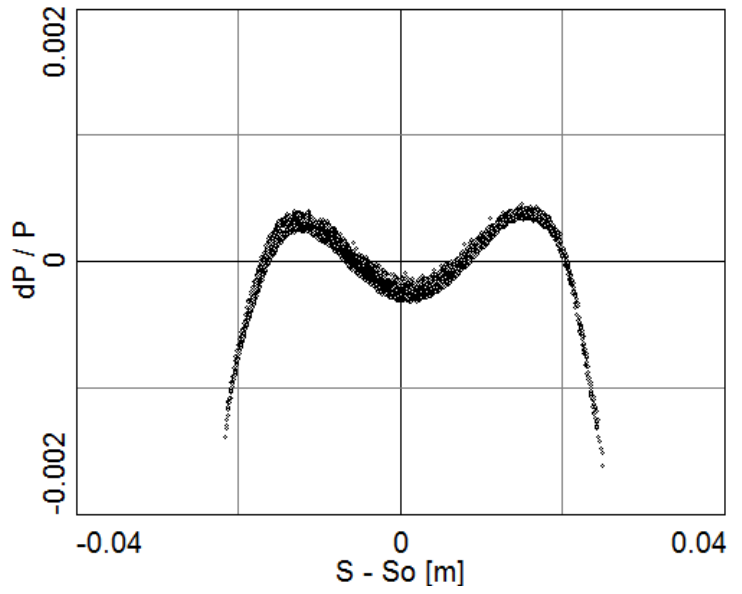

Figure 6: Longitudinal distribution after $4 \mathrm{~km}$ of beam transport using "sliced" approach, for local IBS rates shown in Fig. 5.

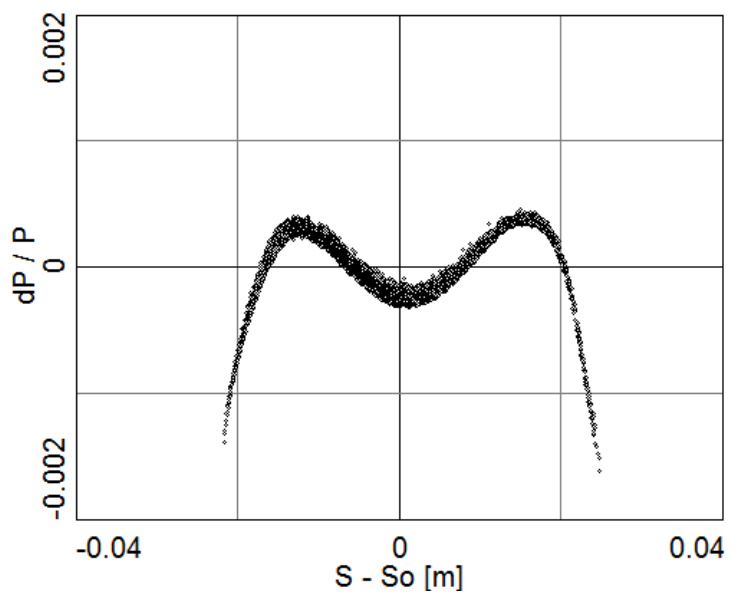

Figure 7: Longitudinal distribution after $4 \mathrm{~km}$ of beam transport using "local" approach, for local IBS rates shown in Fig. 5.
As a next test, we choose beam parameters for which IBS rates calculated based on rms quantities are significant: 1500 1/s and local rates within the slices are very large, as shown in Fig. 8. One can see that only modest change of distribution is observed after $4 \mathrm{~km}$ of beam transport both with the "sliced" and "local" beam approach, as shown in Figs. 9 and 10, respectively.

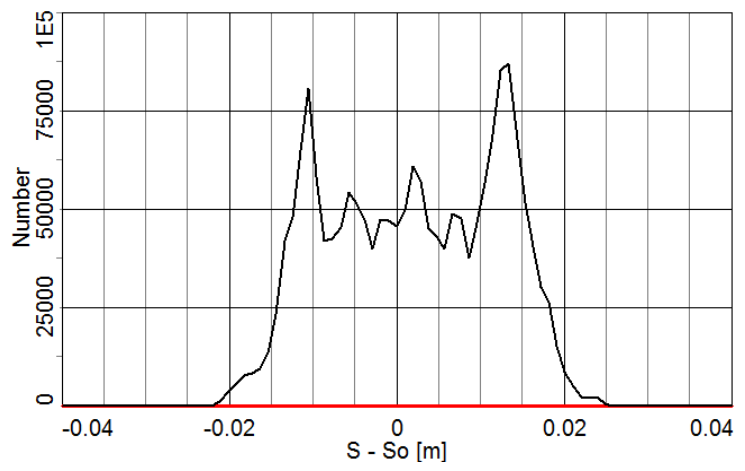

Figure 8: IBS rates within longitudinal slices of beam distribution. Vertical axis: local IBS rates [1/s].

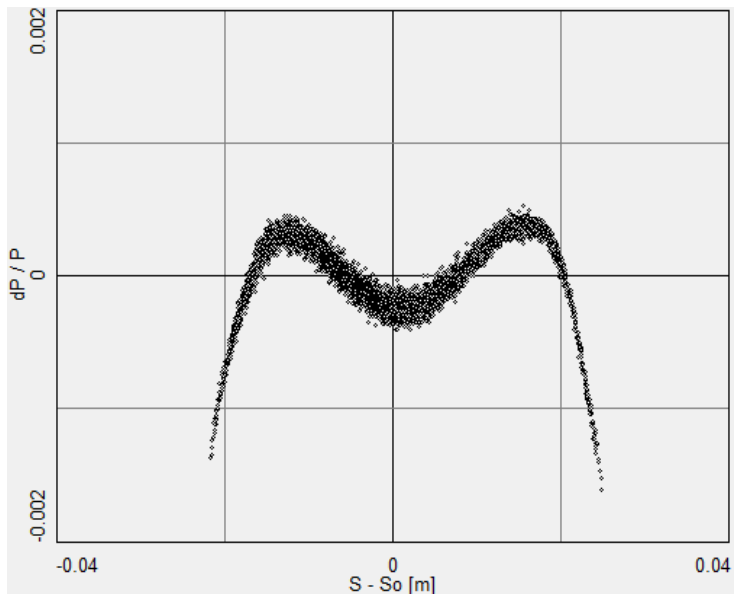

Figure 9: Longitudinal distribution after $4 \mathrm{~km}$ of beam transport using "sliced" approach, for local IBS rates shown in Fig. 8.

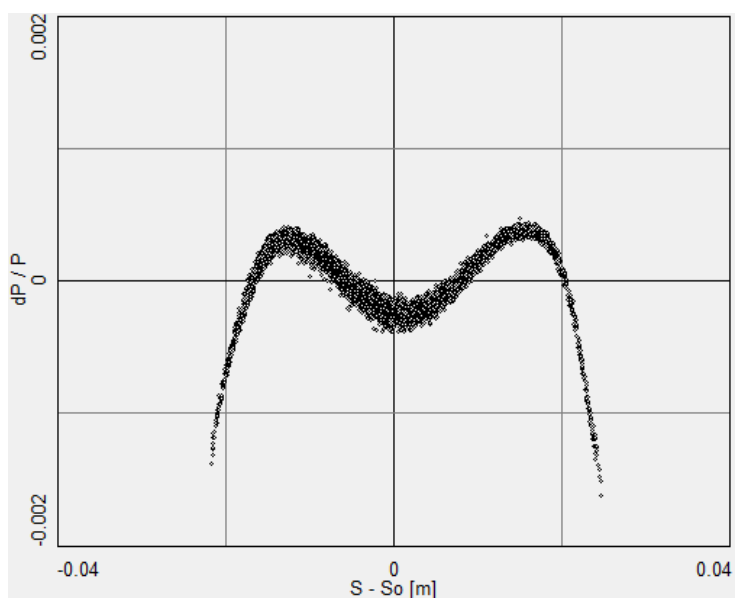

Figure 10: Longitudinal distribution after $4 \mathrm{~km}$ of beam transport using "local" approach, for local IBS rates shown in Fig. 8. 


\section{SUMMARY}

In circular accelerators both the Touschek effect and IBS were found important. The generalized formulas for Touschek calculations are available and are already being used in advanced tracking simulations of several ERLbased projects.

The IBS (which is diffusion due to multiple Coulomb scattering) is not expected to cause any significant effect on beam distribution in ERLs, unless one considers very long transport of high-brightness beams at low energies. Both large and small-angle Coulomb scattering can contribute to halo formation in future ERLs with highbrightness beams, as follows from simple order-ofmagnitude estimates.

In this report, a test comparison between "local" and "sliced" IBS models within the BETACOOL code was presented for an illustrative ERL distribution. We also presented accumulated current loss distribution due to Touschek scattering for design parameters of ERL proposed for the eRHIC project, as well as scaling for multi-pass ERLs.

\section{ACKNOWLEDGMENTS}

We would like to thank D. Kayran, V. Litvinenko, V. Ptitsyn and other members of Accelerator R\&D Division at Collider-Accelerator Department of BNL for useful discussions. Special thanks go to A. Smirnov (JINR, Dubna) who implemented "sliced" approach specifically to enable comparison presented in this report. We also thank members of BETACOOL development team from JINR.

\section{REFERENCES}

[1] C. Bernardini et al., PRL 10, 407 (1963).

[2] H. Bruck and J. Le Duff, Proc. of $5^{\text {th }}$ Int. Conf. on High Energy Accelerators (INFN, Frascati), 1965.

[3] H. Bruck, Circular Particle Accelerators, 1966.

[4] A. Piwinski, Proc. $9^{\text {th }}$ Int. Conf. on High Energy Accelerators, p. 105 (1974); CERN AS 1984, p. 405.

[5] M. Martini, CERN PS/84-9 (1984).

[6] A. Piwinski, CERN AS 1991 (CERN 92-01), p. 226.

[7] Bjorken and Mtingwa, Part. Acc., 13, p.115 (1983).

[8] A.V. Fedotov et al., "Experimental studies of IBS in RHIC and comparison with theories", Proc. of HB2006 Workshop (Tsukuba, Japan, 2006), p. 259.

[9] J. Wei et al., "IBS theory and RHIC experiments", AIP Conf. Proc. 773 (Proc. of HB2004), p. 389.

[10] V. Lebedev, "Single and multiple IBS in hadron colliders", AIP Conf. Proc. 773 (HB2004), p. 440.

[11] A. Burov, "Electron cooling against IBS for highenergy collider", FERMILAB-TM-2213 (2003).

[12] G. Parzen, BNL Tech Note C-AD/AP 144, 150 (2004).

[13] A.V. Fedotov et al., "IBS for distributions under electron cooling", Proc. of PAC05 (Knoxville, TN, 2005), p. 4263.

[14] BETACOOL code : http://betacool.jinr.ru; A. Sidorin et al., NIM A 558, p. 325 (2006).
[15] BNL BETACOOL report, December 2007: http://betacool.jinr.ru/reports/

[16] A.V. Fedotov, A. Sidorin and A. Smirnov, Proc. of HB2010 (Morschach, Switzerland, 2010), p. 62.

[17] A. Xiao and M. Borland, Proc. of PAC09 (Vancouver, Canada), p. 3281.

[18] R.L. Gluckstern and A.V. Fedotov, Phys. Rev. ST Acc. Beams V. 2, 054201, 1999.

[19] N. Pichoff, Proc. of PAC99 (New York), p. 3277.

[20] U. Voelkel, DESY 67/5, 1965.

[21] Y. Miyahara, Jap. Jour. of Appl. Phys. V. 24, No. 9, p. L742 (1985).

[22] A. Piwinski, DESY 98-179, 1998.

[23] A. Xiao and M. Borland, Proc. of PAC07 (Albuquerque, NM, 2007), p. 3453.

[24] G. Hoffstaetter et al., Proc. of EPAC08 (Genoa, Italy), p. 1631.

[25] V. Ptitsyn et al., Proc. of IPAC2011 (Spain), p. 3726.

[26] Sliced-beam approach was implemented in BETACOOL code by A. Smirnov (JINR, Dubna) specifically for benchmarking with "local" IBS model for typical distributions expected in ERLs.

[27] A. Sorensen, Proc. CERN Accelerator School (1987).

[28] A.V. Fedotov et al., Proc. of PAC07 (Albuquerque, NM, 2007), p. 3699. 